\title{
Urogenital Sinus with Mitrofanoff Procedure: Case Review
}

\author{
${ }^{1}$ Child Health Nursing, PSG College of Nursing, Coimbatore, Tamil \\ Nadu, India
}

Jeyamoni David¹ Menaka J' Rajeswari Subramaniam¹ Malarvizhi Gnanam¹

\begin{abstract}
Address for correspondence Malarvizhi G, Child Health Nursing, PSG College of Nursing, Coimbatore, Tamil Nadu, 641004, India (e-mail: malarvizhi2k5@yahoo.co.in).
\end{abstract}

J Health Allied Sci ${ }^{\mathrm{NU}}$ 2019;9:31-34

\begin{abstract}
Keywords

- urogenital sinus

- Mitrofanoff procedure

- clean intermittent catheterization

- urodynamic study

- dimercaptosuccinic acid scan

Urogenital sinus is a rare congenital anomaly. We reviewed a case of urogenital sinus anomaly in 6-year-old girl with recurrent urinary tract infection and small bladder capacity who was referred from another hospital in Coimbatore for further management. The external genitalia appeared normal, and an initial sonogram and repeat micturating cystourethrograms did not indicate any urogenital anomalies. She therefore underwent clean intermittent catheterization. Three years later the child underwent investigations like urodynamic study (UDS) and dimercaptosuccinic acid (DMSA) scan and cystoscopy, followed by ureteric implantation and Mitrofanoff procedure. The presentation of urogenital sinus anomaly with recurrent urinary tract infection is rare and the management is complex.
\end{abstract}

\section{Introduction}

Persistent urogenital sinus (PUGS) is a rare anomaly whereby the urinary and genital tracts fail to separate during embryonic development. It occurs as a result of the arrested migration of the Mullerian ducts from the Muller tubercle to the vestibule. It is a common communication of vagina and urinary tract anywhere from urethral meatus to bladder, but majority from mid to distal urethra. They exit in the perineum as a single opening. Urogenital sinus can occur due to various reasons such as iatrogenic causes, obstructed labor, trauma, and infections. ${ }^{1}$

\section{Case Report}

A six-year-old girl was referred to the surgical OPD of the tertiary care hospital, Coimbatore, in 2017. She had been born full term at 39 weeks of gestation via LSCS (indication: cord around the neck). Till 3 years the child was apparently normal. Later, the child was admitted to the hospital with complaints of fever, poor stream of urine, dribbling of urine, and poor sphincter control, followed by bed wetting for 1 week. After admission detailed history was collected. All blood investigations were done (CBC, biochemistry, serological test, urine routine, and culture sensitivity test). Urodynamic study was planned for the child, but on examination she had a single orifice in the vestibule and hence planned for cystoscopy 9.10.17. It showed single-orifice urogenital sinus with small bladder, and bilateral ectopic ureters and septate vagina. Ureteric reimplantation was done on (October 9, 2017). One month later, the child had fever spikes with urinary tract infection (UTI) and hence cystoscopy and stent removal were performed. She continued to have fever, and the bladder was catheterized, was on continuous drainage, and improved. She was started with antibiotics on II line doxycycllin for 7 days. She was afebrile and discharged. She had regular follow-ups.

One month later again the child got admitted with the complaints of fever, abdominal pain, urgency and frequency of urine, and bedwetting. The child was treated with antibiotic, antipyretics, and vitamin supplementation. Simultaneously, parent counseling was done regarding the bladder augmentation. The child underwent bladder neck sling and augmentation, sigmoid colocystoscopy and appendicular Mitrofanoff surgery on August 20, 2018.

\section{Description of Disease}

In the normal embryological development of the female genitourinary system, at the fourth gestational week the urogenital sinus and rectum enter a common cavity known as the cloaca. At the sixth week of gestation the urorectal septum grows in a caudal direction, separating the cloaca into a ventral urogenital sinus and a dorsal rectum. At the eighth gestational week, a pair of Mullerian ducts develop lateral to the mesonephric ducts, crossing ventromedially 
to fuse in the midline and join the urogenital sinus to form an elevation known as the Mullerian tubercle. Between the 8th and 12th gestational weeks, the Mullerian ducts migrate caudally from the Mullerian tubercle to the vestibule. ${ }^{2}$

Urogenital sinus occurs as a result of the arrested migration of the Mullerian ducts from the Muller tubercle to the vestibule. ${ }^{3}$

\section{Incidence}

It is one of the congenital disorders that are estimated to be 6 in every 100,000 female births.

\section{Clinical Features}

- Fever

- Poor stream of urine

- Dribbling of urine

- Poor sphincter control

- Abdominal pain

- Urgency of urine

- Frequency and bedwetting

- Fatigue

- Sleep disturbance

- Lethargy

- Headache

- Vomiting

\section{Diagnostic Measures}

- History collection.

- Detailed physical examination assessment.

- Laboratory studies of blood (CBC biochemistry, serology).

- Urine routine, culture, and sensitivity.

- USG abdomen.

- Urodynamic study.

- Dimercaptosuccinic acid.

\section{Results}

Urine culture showed pus cells $>25 / \mathrm{HPF}$, bacteria++, colony count $10 \times 5 \mathrm{CFU} / \mathrm{mL}$, organisms: Pseudomonas aeruginosa.

USG ABD: Urinary bladder was moderately distended and showed mild diffuse wall thickening. Floating internal echoes were seen with debris in the dependent part. Postvoidal residual urine volume was $45 \mathrm{~mL}$.

DMSA: Found to have inflammation with decreased function on the right kidney. MCUG was normal.

\section{Management}

Medications prescribed:

Inj. meropenem $280 \mathrm{mg}$ tid $\times 7$ days.

Syp. Crocin $150 \mathrm{mg}$ tid $\times 3$ days.

Syp. Zincovit $5 \mathrm{~mL}$ od (till follow-up).

Tab. Tropan $5 \mathrm{mg} 1$ bd (till follow-up).

\section{Surgical Management}

Mitrofanoff (mi-trof-fan-off) procedure was created by Professor Paul in 1976. The Mitrofanoff procedure, also known as the Mitrofanoff appendicovesicostomy, is a surgical proce- dure in which the appendix is used to create a conduit between the skin surface and the urinary bladder. A small channel (that connects the bladder to the outside of the body) and the catheter are used to empty urine from the bladder through the channel, and a one-way flap valve is used to maintain urine control. ${ }^{4}$

\section{How Does Mitrofanoff Work?}

- As the bladder fills, urine pressure builds up and helps to compress the tunneled channel.

\section{Construction of Mitrofanoff Procedure}

- To create a Mitrofanoff, under the general anesthesia the surgeon makes a narrow tube using a piece of appendix ( - Fig. 1).

- In case appendix is absent in the patient, small bowel will be used.

- The narrow tube is sewn to skin. The opening is called a stoma, and it is usually placed in the belly button, making it fairly inconspicuous.

- The other end of the narrow tube is connected to the bladder (reservoir) using a tunneling technique to create a flap valve (-Fig. 2).

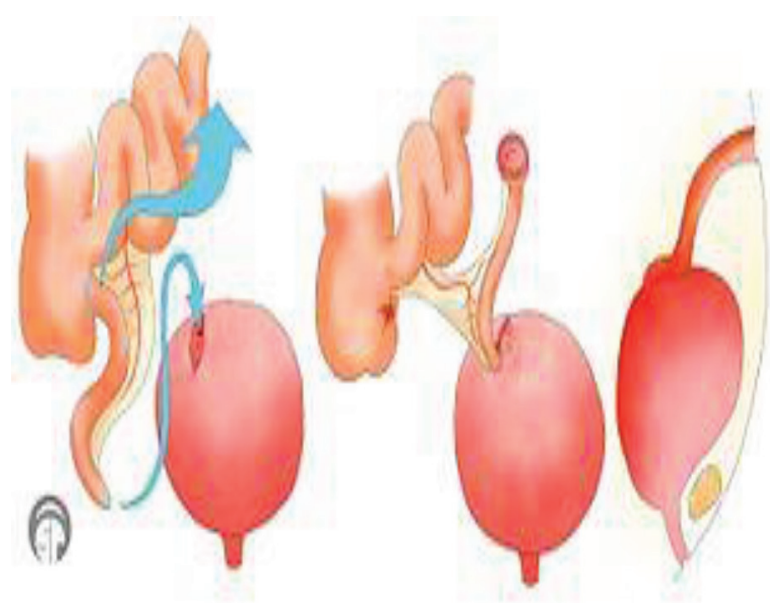

Fig. 1 Mitrofanoff appendicovesicostomy https://en.m.wikipedia.org/ wiki/Mitrofanoff_procedure

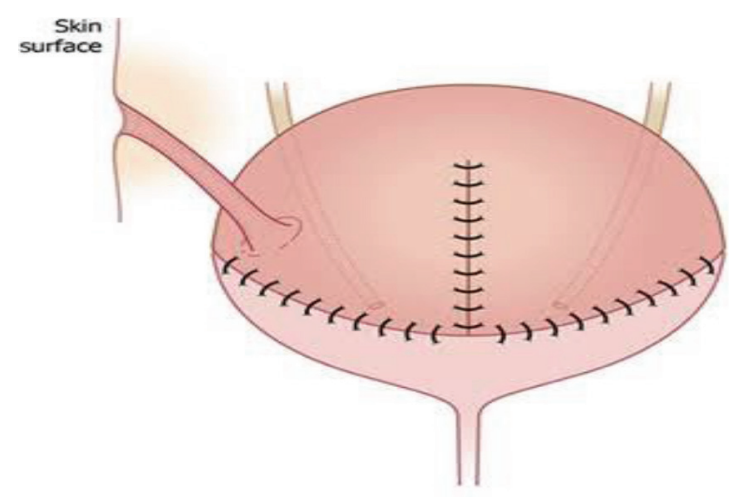

Fig. 2 Continent, catheterizable Mitrofanoff channel created from isolated appendix or segment of small intestine. 


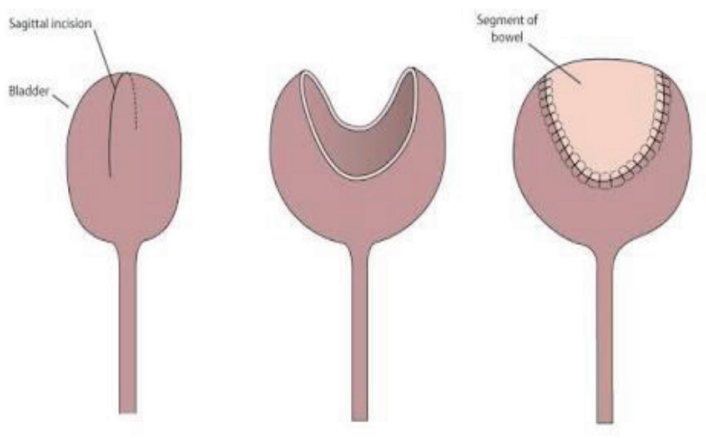

Fig. 3 Bladder augmentation.

- As the bladder pressure rises, the tunneled channels become compressed against the wall of the bladder, creating a one-way valve that prevents urine leakage.

- To drain the bladder, a catheter is passed 4 to 5 times a day through the one-way flap valve.

- Once the bladder is drained (this usually takes a couple of minutes), the catheter is removed.

\section{Bladder Augmentation}

Bladder augmentation is a surgical alteration of the urinary bladder. It involves removing strips of tissue from the intestinal tract and adding this to the tissue of the bladder. ${ }^{1}$ This has two intended results: increased bladder volume and a reduced percentage of the bladder involved in contraction that in turn results in lower internal pressures in the bladder during urination ${ }^{5}$ (-Fig. 3 ).

\section{Discussion}

The six-year-old girl was on follow-up for urogenital sinus with bilateral ectopic ureters. She had bilateral ectopic ureters with recurrent UTI and hence bilateral uretereic reimplantation was done and treated with tropan. The most distressing complaint in the child was urinary incontinence and UTI which made her a social outcaste, mostly unable to attend school. In this case bladder augmentation and Mitrofanoff were a tremendous boost in the psychosocial life of the child and her family. In addition, bladder augmentation with Mitrofanoff procedure resulted in long-term benefits in terms of resolution or stabilization of the upper tract pathology unless renal failure advanced. However, it incurs necessary changes in lifestyle, such as need for clean intermittent catheterization (CIC) and bladder washouts, and also has inherent long-term complications. Thus, the place of bladder augmentation in pediatric reconstructive urology needs a thorough understanding of the common conditions where it might be indicated.

This present condition concurs with another cohort study conducted among 300 children admitted for neurogenic bladder management in the Christian Medical College, Vellore. Seventy-eight children had undergone bladder augmentation. Among 78 newborns, 71 cases had complaints of bladder associated with upper tract changes. The other seven cases underwent Bladder Augumentation for intractable incontinence with normal upper tract. Bladder neck surgery to tighten or even close the bladder neck was performed with bladder augmentation. CIC becomes universally practiced in the management of newborn. ${ }^{6}$

\section{Complications}

- Risk of infection.

- Stoma at the skin can become narrow.

- Difficult catheterization.

- Leakage through the channel.

\section{Nursing Management}

Preoperative Care

- Explain the procedure to the parents and the need of surgery.

- Informed written consent to be obtained.

- Complete blood investigations including biochemistry and serology test to be done.

- Maintain the NPO status.

- Anesthetist's opinion to be obtained and performed.

- Provide adequate psychological support.

- Ensure all the preoperative orders have been performed.

\section{Postoperative Care}

Pain

- Administer the analgesic as per the physician's advice.

\section{Diet}

- Keep NPO for 4 to 5 days.

- Keep NG tube in place.

- Start clear liquids after passing the stool.

- Check the sign of vomiting.

- Maintain fluid management.

\section{Catheter Care}

- Check daily that the catheter draining is normal.

- Follow aseptic technique.

- Keep the skin clean and dry.

- Teach the parents how to pass a catheter in and out to empty the bladder/neo bladder.

- Educate the parents how to flush the catheter, to be continued until the return fluid is clear.

- If the catheter gets blocked with mucus plugs, these must be plugged out and unblocked as soon as possible.

- Notice the color of urine.

- Advise the patient to take rest at least for 6 weeks.

- Advise the parents to immediately contact the pediatric surgery department if they find difficulties with this at home.

- Follow-up appointment is necessary. 


\section{Key Points}

- The Mitrofanoff procedure creates a channel between the bladder and the skin of abdominal wall.

- The small tube (catheter) could be passed through the channel to empty the bladder.

- The channel can be reframed as avoids the need for a permanant bag.

- It is often performed with either entrocystoplasty (enlargement of bladder with a bowel patch) or radical cystectomy with a new bladder made from a bowel.

- A Mitrofanoff channel is prone to technical problems (e.g., leaking of urine or narrowing) that often require revision surgery.

\section{Conclusions}

The augmentation and Mitrofanoff procedure provide an adequate, safe, and low-pressure reservoir of urine except in cases of bladder exstrophy and previous abdominal surgery. It is feasible in a developing country if the family is motivated. When done for the correct indication with meticulous preoperative assessment and postoperative care, it can make a great improvement in the quality of life and provide long-term renal protection. Parent and child were trained on bladder wash after 15th postoperative day of discharge. Both were able to perform CIC and bladder wash. There was no postoperative infection and the hospitalization was uneventful.

\section{Conflict of Interest}

None declared.

\section{References}

1 Ludwikowski BM, González R. The surgical correction of urogenital sinus in patients with DSD: 15 years after description of total urogenital mobilization in children. Pediatr Surg. 2010;42:198-201

2 Tan HH, Tan SK, Shunmugan R, Zakaria R, Zahari Z. A case of persistent urogenital sinus: pitfalls and challenges in diagnosis. Sultan Qaboos Univ Med J 2017;17(4):e455-e459

3 Singh S, Singh P, Singh RJ. Persistent urogenital sinus. J Anat Soc India 2010;59:242-244

4 Bischoff A. The surgical treatment of cloaca. Semin Pediatr Surg 2016;25(2):102-107

5 Soygur T, Burgu B, Zümrütbas A, Süer E. The need for ureteric re-implantation during augmentation cystoplasty: video-urodynamic evaluation. BJU Int 2010;105(4):530-532

6 Sen S, Chacko J, Karl S. Pediatric bladder augmentation in developing country: lessons learnt from an experience of 195 cases. JIMSA 2014;27(2):79-80 